\title{
Interactive comment on "The role of air-sea fluxes for the water vapour isotope signals in the cold and warm sectors of extratropical cyclones over the Southern Ocean" by Iris Thurnherr et al.
}

\section{Anonymous Referee \#1}

Received and published: 27 October 2020

Please see attached pdf.

Please also note the supplement to this comment:

https://wcd.copernicus.org/preprints/wcd-2020-46/wcd-2020-46-RC1-supplement.pdf

Interactive comment on Weather Clim. Dynam. Discuss., https://doi.org/10.5194/wcd-2020-46, 2020. 